\title{
USO DAS TIC ASSOCIADAS A METODOLOGIAS ATIVAS EM CONTEXTO UNIVERSITÁRIO
}

\author{
THE APPLICATION OF ICT'S ASSOCIATED WITH ACTIVE LEARNING IN THE UNIVERSITY CONTEXT \\ USO DE LAS TIC ASOCIADAS A METODOLOGÍAS ACTIVAS EN EL CONTEXTO UNIVERSITARIO
}

\author{
Asafe Costa Lopes \\ Doutorando em Biociências Aplicadas a Saúde - Universidade Federal de Alfenas (UNIFAL/MG). Especialista \\ em Mídias na Educação - Universidade Federal de São João del Rei (UFSJ) \\ E-mail: asafecosta@gmail.com
}

Héber Sorares Caixeta

Mestre em Ciência e Engenharia Ambiental - Universidade Federal de Alfenas /MG Especialista em Mídias na Educação. Universidade Federal de São João del Rei (UFSJ).

E-mail: heber_caixeta@yahoo.com.br

\begin{abstract}
RESUMO
O presente trabalho avaliou, via questionário anônimo, 42 alunos de um curso da área da saúde de uma instituição de ensino particular do Sul de Minas Gerais acerca de suas impressões sobre a renovação das metodologias de ensino com a utilização de tecnologias e metodologias ativas em sala de aula ao longo do primeiro semestre de 2018 e o seu impacto no aprendizado estudantil. Com base nos dados obtidos, conclui-se que as metodologias inovadoras associadas a tecnologias educacionais, têm o poder de transformar a educação contemporânea fazendo com que alunos não adaptados aos modelos tradicionais de ensino, possam compreender melhor as disciplinas ministradas e visualizar suas aplicabilidades e importância no contexto social. Verifica-se que ainda há um longo caminho a ser percorrido uma vez que a capacitação docente e a infraestrutura necessária para o emprego de tais técnicas ainda são deficientes, apesar de que grande parte dos alunos estejam receptivos e conscientes da necessidade destas mudanças.
\end{abstract}

Palavras-chave: Metodologias Inovadoras; Tecnologia; Educação; Aprendizado.

\begin{abstract}
The aim of this work was evaluating with anonymous questionnaire, 42 students of a health graduating students of a particular institution in South of the State of Minas Gerais, Brazil, about your impressions of the adaptation of traditional learning improving it with technology and active learning during the first semester of 2018 and the impact of these in their learning. In brief, we could conclude that the innovative methodologies associated with technology have the power of changes the contemporary education, making that students that do not fit in the traditional method of learning understand better the contents and see his applicability's and importance in the social context. It is visible that there is a long way to go, because the professors are often unprepared and due to the lack of infrastructure needed to apply these techniques, despite of a great part of the students are receptive and conscious about the necessity of this changes.
\end{abstract}

Keywords: Active-learning. Technology. Education. Learning.

\section{RESUMEN}

El objetivo de este trabajo fue evaluar, por medio de un cuestionario anónimo, a 42 estudiantes graduados en salud de una institución privada en el sur del estado de Minas Gerais, Brasil, acerca de sus impresiones sobre la renovación de los métodos de enseñanza tradicionales con el uso de tecnologías y metodologías activas durante el primer semestre de 2018 y su impacto sobre el aprendizaje. En resumen, podemos concluir que las metodologías innovadoras asociadas con las tecnologías tienen el poder de cambiar la educación contemporánea, haciendo que los estudiantes que no encajen en el método tradicional de aprendizaje comprendan 
mejor los contenidos y vean su aplicabilidad e importancia en el contexto social. Es visible que hay un largo camino por recorrer, una vez que los profesores a menudo no están preparados y debido a la carencia de infraestructura necesaria para aplicar estas técnicas, a pesar de que gran parte de los estudiantes está receptiva y consciente de la necesidad de estos cambios.

Palabras-clave: Metodologías Activas. Tecnología. Educación. Aprendizaje.

\section{INTRODUÇÃO}

A partir da geração $X$, a tecnologia se tornou parte integral do cotidiano das novas gerações, o que leva ao surgimento da geração $\mathrm{Y}$, mais atuante no contexto atual da sociedade, e que convive com a modificação da relação do homem com o tempo através da instantaneidade das coisas, como por exemplo, a comunicação e o rápido acesso a informações variadas por meio da Internet. Conforme Kuntz (apud DE MALAFAIA, 2011), dentre suas características diversas, destacam-se a autoconfiança, o sucesso financeiro, além de serem bastante informais, agitados, ansiosos e imediatistas.

A medida que novas tecnologias surgem e se tornam disponíveis para a população, os métodos tradicionais de ensino, no qual o professor é o centro das atenções, e por meio da lousa expõe o conteúdo, vêm se tornando ineficientes. Os professores atuais enfrentam o grande desafio de competir com os atrativos smartphones dos alunos que cada vez mais dominam o ambiente escolar (BASTOS, 2015; BAKIR, 2016).

Visando conter a desatenção dos alunos por estarem conectados a "outros mundos" durante a aula e atendendo ao novo perfil do jovem, as metodologias inovadoras de ensino têm se tornado cada vez mais relevantes no contexto escolar. Fazem do aluno o protagonista do seu aprendizado e exigem cada vez mais que este se dedique e busque conhecimento nas mais variadas fontes, o que transforma o professor em um direcionador do aprendizado e complementador de conteúdo (FREEMAN et al., 2014; ROACH, 2014; PARMELEE, AL-KADI, 2014; CAMARGO, PITAGUARI , DALBERTO, 2017; MACEIRA, DANITTA, 2017).

Concomitante a isso, a utilização das tecnologias da informação no ambiente escolar vem adquirindo cada vez mais relevância, pois possibilita aulas mais dinâmicas e permite apresentar conteúdos de formas diferenciadas, capazes de prender a atenção do aluno; utiliza-se inclusive o temido smartphone como uma ferramenta educacional excelente e 
com diversas possibilidades (HOLLAND, HOLLAND, 2014; BULMAN, FAIRLIE, 2016).

Tais mudanças levam os educadores a se enfrentarem com constantes readaptações a fim de que a forma do processo de ensino/aprendizagem ocorra de acordo com o novo perfil dos jovens. Observa-se uma grande discrepância entre gerações, o que produz a necessidade de uma maior readaptação do contexto educacional para que os novos estudantes, como os da geração $\mathrm{Y}$, possam desfrutar de ambientes escolares que supram suas necessidades de imediatismo e autoconfiança, já quase totalmente incompatíveis com o tradicional quadro negro e giz (EZENWABASILI, 2016).

Neste contexto, é extremamente importante avaliar se a utilização de tais conteúdos refletem realmente em um aprimoramento do processo ensino/aprendizagem uma vez que existe uma tênue linha entre a utilização efetiva de ferramentas que garantam um melhor aprendizado, e a sua utilização com finalidades diversas como marketing educacional ou o preenchimento de lacunas deixadas por planos de ensino pobres em conteúdo e que não atendem às demandas reais do mercado de trabalho atual (MOLESWORTH, NIXON, SCULLION, 2009; BULMAN, FAIRLIE, 2016).

Ressalta-se ainda a importância da verificação da disponibilidade de infraestrutura dos ambientes educacionais brasileiros para a aplicação de tais recursos, uma vez que grande parte destes requerem como pré-requisito básico a disponibilidade de Internet de velocidade razoável e acessível aos discentes para a realização de atividades em ambiente escolar, o que pode inviabilizar a inserção das tecnologias e o suprimento das demandas exigidas pela geração de estudantes atuais (SÁTYRO, SOARES, 2007; GARNICA, TORKOMIAN, 2009; SOARES et al, 2013).

Com o surgimento da possibilidade do uso da tecnologia no âmbito educacional, novos desafios foram criados. Dentre os grandes problemas encontrados estão a falta de preparo e de cursos de aprimoramento para docentes que se disponham a empregar novas metodologias em suas disciplinas, bem como a infraestrutura não adaptada a essa nova realidade. A utilização de metodologias inovadoras de ensino com o uso da tecnologia é um processo que não depende somente do professor, que necessita da participação ativa do discente que, se não estiver aberto a essa nova realidade de ensino, torna o processo 
ineficaz.

Apesar dos grandes desafios, acredita-se que os alunos são bastante receptivos à utilização das metodologias inovadoras e das TIC e que estas podem melhorar a qualidade do ensino e da aprendizagem por tornar o ensino mais visual, tátil e aplicado, aliado ao seu contato cotidiano com a tecnologia. No entanto, em alguns momentos a infraestrutura inadequada, bem como o despreparo do professor, torna sua aplicação inviável ou não aproveitada em sua totalidade.

Entendendo que o mundo sofre mudanças constantes, o presente trabalho tem por objetivo avaliar, via questionário anônimo, 42 alunos de um curso da área da saúde de uma instituição de ensino particular do Sul de Minas Gerais acerca de suas impressões sobre a renovação das metodologias de ensino por meio da utilização de tecnologias e metodologias ativas em sala de aula, ao longo do primeiro semestre de 2018 e o seu impacto no aprendizado estudantil, identificando fatores como receptividade, primeiras impressões e eficácia do método.

\section{Metodologia}

Realizou-se um levantamento, via questionário anônimo, acerca das impressões dos alunos com relação à utilização dos recursos tecnológicos e de metodologias inovadoras em sala de aula. Para tanto, alunos do curso de Graduação em Biomedicina de uma instituição particular do Sul de Minas Gerais foram submetidos a aulas teóricas com metodologias inovadoras associadas a recursos tecnológicos, como vídeos, quizzes online e presenciais, ao longo de um semestre. Posteriormente, responderam ao questionário para a verificação de informações como a melhoria no entendimento do conteúdo, qualidade de condução e execução das técnicas pelos professores, readequações de infraestrutura necessárias para um bom funcionamento metodológico, bem como a sua preferência pelo método convencional frente às novas metodologias. O grupo de estudo foi composto por 42 alunos, 22 ingressantes e 20 já no segundo ano de curso, o que permitiu verificar se os discentes já haviam tido algum contato com tais metodologias fora do contexto univer- 
sitário, bem como perceber a diferença entre aulas expositivas ministradas versus aulas inovadoras pelos alunos veteranos.

\title{
Resultados
}

A primeira pergunta respondida pelo grupo estudado foi acerca do seu conhecimento prévio a respeito das tecnologias inovadoras de ensino.

Conforme observado (Tabelas 01 e 02), grande parte dos alunos do terceiro período, presentes há mais de um ano na instituição, não conheciam as metodologias ativas de ensino, ao contrário, grande parte dos alunos ingressantes já haviam vivenciado essa realidade ou ouviram falar a respeito dela. Isso, apesar de a Lei de Diretrizes e Bases da Educação conter artigo aconselhando o seu uso.

\begin{abstract}
A Lei de Diretrizes e Bases da Educação, № 9.394, de 20 de dezembro de 1996, em seu artigo 35, parágrafo 8 , determina a utilização de atividades teóricas, práticas, provas orais e escritas, seminários, projetos e atividades on-line, possibilitando ao término do ensino médio que o estudante, de acordo com o inciso número um, possuir domínio dos princípios científicos e tecnológicos que presidem a produção moderna.
\end{abstract}

A lei supracitada ressalta a importância e a necessidade de maior aplicação de tais técnicas no ensino médio, com a finalidade de que os alunos ingressantes no ensino superior já possuam as habilidades e competências necessárias para esse novo modelo de educação que cada dia mais vem sendo empregado pelas instituições universitárias (DELPHINO et al. 2017).

Apesar da proibição legal da utilização de celulares em sala de aula, deve-se ter em mente que este já é parte indispensável do cotidiano da grande maioria dos alunos e combatê-lo não é a melhor opção a ser adotada. A instituição tem que aventurar-se a adotar o telefone celular dos alunos como ferramenta educacional, criar políticas combativas a desigualdades na utilização do telefone celular e nas novas tecnologias entre docentes e alunos (ROCHA, LISBOA, 2016). A escola deve contemplar em seu planejamento a utilização das tecnologias digitais disponíveis, de fácil aquisição e maior popularidade, como o telefone celular (TIMBANE, AXT, ALVES, 2015). Com relação à utilização do celular como ferramenta de ensino, nota-se que a grande maioria dos estudantes concordam que os 
recursos tecnológicos oferecidos hoje por tais dispositivos eletrônicos podem contribuir para o seu aprendizado acadêmico, comprovando que a utilização do telefone celular em ambiente educacional pode possibilitar desenvolvimento intelectual e social dos alunos, se bem empregado (ANSHARI et al, 2017; VIEIRA, ALVES, 2015).

Tabela 01: Resultados obtidos via questionário anônimo aplicado a 22 alunos ingressantes e 20 veteranos ( $3^{\circ}$ Período). Os resultados estão apresentados em número absoluto de respostas obtidas em cada questão.

\begin{tabular}{|c|c|c|}
\hline \multicolumn{3}{|c|}{ RESULTADOS OBTIDOS } \\
\hline \multicolumn{3}{|c|}{ CONHECIMENTO DAS METODOLOGIAS ATIVAS } \\
\hline Respostas & $1^{\circ}$ Período $(\mathrm{N}=22)$ & $3^{\circ}$ Período $(\mathrm{N}=20)$ \\
\hline
\end{tabular}

Foram utilizadas com outros nomes

4

Conhecia, mas não utilizou em aula

Foram utilizadas no ensino médio/pré-vestibular

Não conhecia

7

11

O CELULAR PODE SER UTILIZADO COMO FERRAMENTA DE APRENDIZADO?

\begin{tabular}{|c|c|c|}
\hline Sim & 20 & 19 \\
\hline Não & 2 & 1 \\
\hline \multicolumn{3}{|c|}{ PRIMEIRO CONTATO COM AS METODOLOGIAS ATIVAS } \\
\hline Achou que o professor estava enrolando a aula & 0 & 0 \\
\hline Achou complexo e difícil de entender & 1 & 3 \\
\hline Não achou uma forma ideal de ensinar & 0 & 3 \\
\hline Gostou da utilização das técnicas & 21 & 15 \\
\hline \multicolumn{3}{|c|}{ COMO OS ALUNOS APRENDERAM MAIS } \\
\hline Metodologias inovadoras & 18 & 12 \\
\hline Aulas Expositivas & 2 & 5 \\
\hline Apresentação de Seminário & 1 & 0 \\
\hline Sozinhos & 1 & 3 \\
\hline \multicolumn{3}{|c|}{$\begin{array}{l}\text { DIFICULDADES ENCONTRADAS PELOS PROFESSORES NA APLICAÇÃO DAS METO- } \\
\text { DOLOGIAS }\end{array}$} \\
\hline Não explicou corretamente o procedimento & 1 & 5 \\
\hline Não houve objetivos definidos & 1 & 3 \\
\hline Dificuldade na utilização da tecnologia & 20 & 6 \\
\hline Desmotivação & 0 & 4 \\
\hline \multicolumn{3}{|c|}{ TODOS OS PROFESSORES ESTÃO APTOS À REALIZAÇÃO DAS TÉCNICAS? } \\
\hline Sim & 13 & 13 \\
\hline Não & 9 & 7 \\
\hline \multicolumn{3}{|c|}{$\begin{array}{l}\text { FREQUENCIA EM QUE DEVERIAM SER EMPREGADAS AS METODOLOGIAS INOVADO- } \\
\text { RAS }\end{array}$} \\
\hline Semestral & 0 & 5 \\
\hline Mensal & 1 & 8 \\
\hline Quinzenal & 14 & 6 \\
\hline Todas as Aulas & 7 & 1 \\
\hline
\end{tabular}


Fonte: Os autores (2018).

A grande maioria dos estudantes questionados demonstraram apreço pela utilização de formas diferentes de ensino, sendo que os alunos do primeiro período, que já tiveram algum contato com o modelo de ensino, demonstram a vontade de maior utilização das mesmas. Observou-se, porém, maior resistência de alunos já adaptados ao modelo tradicional de ensino. Tais informações corroboram com estudos anteriores que relatam a eficácia da utilização das metodologias ativas em reduzir o índice de reprovação e aumentar o engajamento dos alunos com o conteúdo (PERES; SUZUKI, AZEVEDO-MARQUES; 2015; FREEMAN et al, 2014). A grande preocupação da maioria dos docentes é que, ao utilizar a metodologia de ensino, os alunos possam interpretar como uma forma de engodo para que o tempo de aula possa ser preenchido, fato não observado no grupo estudado. Ressalta-se a importância da observância de critérios e orientações bem estabelecidos ao utilizar tais técnicas a fim de que os alunos compreendam de forma integral como acontecerá o processo de aprendizagem (BAEPLER; WALKER; DRIESSEN, 2014), sem descuidar a utilização de aulas expositivas, a fim de que o aluno mais passivo também possa ser atendido (TSENG et al. 2016).

Como citado anteriormente, a aplicação de metodologias inovadoras e recursos tecnológicos em sala de aula tem a capacidade de ampliar o aprendizado dos alunos por explorar a argumentação e discussão das temáticas abordadas (ABDI, 2014). Fato similar foi encontrado nos dados obtidos por meio de questionário, onde observou-se que grande parte dos alunos considera ter aprendido melhor o conteúdo por meio das técnicas de metodologias ativas de ensino e aplicativos educacionais do que através de outras formas de ensino, como as aulas expositivas e o autodidatismo.

A impressão dos alunos acerca da forma de emprego das metodologias por parte dos professores é bastante variada. Para os alunos do primeiro período, a maior dificuldade foi a utilização da tecnologia, fator limitante para um bom emprego de algumas metodologias ativas e determinante no uso das TIC (EICKHOLT, 2018). Ressalta-se então a importância de programas de capacitação docente a fim de que estes estejam mais preparados e aptos para esta nova realidade, presente no contexto educacional atual (LÓPEZ, TRON- 
CON, 2015). As impressões de alunos do terceiro período refletem um maior conhecimento e convívio com seus docentes; indicaram que vários fatores foram limitantes ao emprego das tecnologias e metodologias. Percebem o anseio por parte dos docentes pelo emprego das mesmas e ao mesmo tempo o seu despreparo. Esse fato $r$ explica a percepção de desmotivação por parte dos alunos, e evidencia a necessidade de maiores esforços por parte da instituição de ensino com o intuito de promover capacitação docente e conscientização da importância do seu papel no ambiente educacional. Dados similares foram encontrados em estudo realizado por Eickholt (2018), onde a falta de incentivo por parte da instituição, o grande tempo gasto no preparo dos novos conteúdos, bem como a impressão de que tais técnicas são desnecessárias, foram fatores limitantes na mudança no estilo de ensino, corroborando com os resultados obtidos onde parte dos alunos considera que nem todos os professores estão aptos para o emprego de formas diferenciadas de ensinar.

TABELA 02: Resultados obtidos via questionário anônimo aplicado a 22 alunos ingressantes e 20 veteranos ( $3^{\circ}$ Período). Os resultados estão apresentados em número absoluto de respostas obtidas em cada questão.

\section{RESULTADOS OBTIDOS}

OPINIÃO A RESPEITO DA UTILIZAÇÃO DE VÍDEOS DURANTE AS AULAS EXPOSITIVAS

\begin{tabular}{lcc}
\hline Respostas & $\mathbf{1}^{\mathbf{0}}$ Período (N=22) & $\mathbf{3}^{\circ}$ Período (N=20) \\
\hline Desnecessária & 1 & 0 \\
Ajuda parcialmente na compreensão & 5 & 7 \\
Facilita o entendimento & 16 & 12 \\
Os professores não escolhem o vídeo correto & 0 & 1 \\
\hline
\end{tabular}

\begin{tabular}{lcc}
\hline \multicolumn{3}{c}{ SATISFAÇÃO QUANTO À UTILIZAÇÃO DE METODOLOGIAS INOVADORAS } \\
\hline Recomendariam a utilização a outras pessoas & 16 & 14 \\
Insatisfeitos & 5 & 5 \\
Não recomendariam & 1 & 1 \\
\hline \multicolumn{3}{c}{ PROBLEMAS DE INFRAESTRUTURA MAIS COMPROMETEDORES } \\
Datashow & 1 & 1 \\
Wifi & 21 & 19 \\
\hline
\end{tabular}

Fonte: Os autores (2018).

Os alunos ainda foram interrogados com relação à utilização de vídeos durante as aulas expositivas. Grande parte dos alunos concordou que a exibição de vídeos correlatos ao assunto estudado pode facilitar e ajudar parcialmente na compreensão do conteúdo ministrado, corroborando o que foi observado por Dale (1969), quem indica que os alunos 
são capazes de absorver cerca de $20 \%$ do conteúdo aprendido através de recursos audiovisuais.

Perguntou-se, ainda, sobre o grau de satisfação e se os alunos recomendariam uma instituição de ensino preocupada pelo emprego de metodologias inovadoras de ensino. Observou-se que grande parte dos alunos estão satisfeitos e recomendariam a utilização de tais técnicas, fato que pode ser justificado pelo aumento do dinamismo e possibilidade de participação mais ativa dos estudantes durante aulas, além da possibilidade de apresentação dos conteúdos de forma mais atrativa (LOBO, MAIA, 2015).

Uma das grandes dificuldades encontradas na aplicação das tecnologias educacionais é a infraestrutura de rede, na qual a Internet sem fio nem sempre funciona como o esperado. A instituição deve estar preparada neste sentido antes do emprego das metodologias, uma vez que há grande prejuízo no aprendizado quando as metodologias não funcionam devido à infraestrutura necessária (COCA, SLISKO, 2013; EICKHOLT, 2018). Em estudo realizado por Souza e colaboradores (2017), apesar da existência de computadores disponíveis aos alunos nas escolas brasileiras, a quantidade é insuficiente, considerando o número de alunos por máquina. Ainda segundo os autores, o acesso à Internet via smartphones corresponde a cerca de $39 \%$ do total de acessos à Internet dentro das escolas brasileiras para realização de pesquisas, trocas de mensagens de textos e acesso a mídias sociais, demonstrando assim a grande necessidade de maiores investimentos em infraestrutura de Internet para que o telefone celular dos estudantes possa ser melhor empregado em metodologias educacionais. Fato similar foi encontrado no presente estudo, onde 92\% dos estudantes pesquisados indicaram que há falta de infraestrutura necessária para a utilização das tecnologias.

\section{CONSIDERAÇÕES FINAIS}

Verifica-se assim, a indissociabilidade entre tecnologia e educação em um mundo cada vez mais globalizado. Para que tal emprego ocorra de forma saudável é importante que gestores e professores trabalhem em sintonia, havendo a percepção da importância 
do emprego de metodologias diferenciadas, bem como de constante aprimoramento docente por meio de cursos de atualização que possam ser realizados de forma frequente, a fim de que todos possam estar a par das aplicações educacionais disponíveis, bem como aptos para a sua utilização.

Ressalta-se ainda que de nada adianta uma instituição investir em capacitação docente se não houver concomitantemente empenho por parte das secretarias de educação e governos estaduais e federais na melhoria da infraestrutura escolar no que diz respeito ao fornecimento de conexão de Internet com velocidade adequada para a sua utilização em sala de aula, além de dispositivos compartilháveis para alunos que não possuam recursos financeiros para adquiri-los.

Conclui-se que as metodologias inovadoras associadas a tecnologias educacionais, têm o poder de transformar a educação contemporânea, fazendo com que alunos já não mais adaptados aos modelos tradicionais de ensino, possam compreender melhor as disciplinas ministradas e visualizar sua aplicabilidade e importância no contexto social. Verifica-se que ainda há um longo caminho a ser percorrido, uma vez que a capacitação docente e a infraestrutura necessária para o emprego de tais técnicas ainda são deficientes, apesar de grande parte dos alunos estarem receptivos e conscientes da necessidade destas mudanças.

\section{REFERÊNCIAS}

ABDI, Ali. The effect of inquiry-based learning method on student's academic achievement in science course. Universal Journal of Educational Research. V.2, n.1, p. 37-41, 2014.

ANSHARI, M. et al. Smartphones usage in the classrooms: Learning aid or interference? Education and Information Technologies, v. 22, n. 6, p. 3063-3079, 19 jan. 2017.

BAEPLER, Paul; WALKER, J. D; DRIESSEN, Michelle. It's not about seat time: blending, flipping and efficiency in active learning classrooms. Computers \& Education. V.17, p.227-236, 2014. 
BAKIR, N. Technology and Teacher Education: A Brief Glimpse of the Research and Practice that Have Shaped the Field. TechTrends, v. 60, n. 1, p. 21-29, jan. 2016.

BASTOS, João Augusto. A educação tecnológica-conceitos, características e perspectivas. Revista Educação \& Tecnologia, v. 1, n. 1, 2015.

BRASIL. LEI N 9.394, de 20 de dezembro de 1996.Diretrizes e Bases da Educação Nacional. Brasília, DF, dezembro de 2016. Disponível em: http://www.planalto.gov.br/CCIVIL_03/ Leis/L9394.htm. Acesso em: 27 de ago. 2018.

BULMAN, G.; FAIRLIE, R. W. Technology and Education. In: Handbook of the Economics of Education. Elsevier, p. 239-280, 2016.

CAMARGO, Fausto; PITAGUARI, Antônio; DALBERTO, Dirce Maria. O Uso do Team-Based Learning como Estratégia de Avaliação Formativa no Curso de Administração da Uniamérica. Revista Pleiade, v.11, n.21, p.77-89, 2017.

COCA, David Mendez \& SLISKO, Josip. Software socrative and smartphones as tools for implementation of basic processes of active physics learning in classroom: an initial feasibility study with prospective teachers. European Journal of Physics Education. V.4, n.2, p.17-24, 2013.

DALE, Edgar. Edition of Audio-visual methods in teaching. 3ed New York: Dryden Press, 1969.

DE MALAFAIA, Gabriela Silva. Gestão estratégica de pessoas em ambientes multigeracionais. Disponível em: http://www.inovarse.org/sites/default/files/T11_0452_2151.pdf. Acesso em: 29 ago.2018.

DELPHINO, Fátima Beatriz de Benedictis. O papel das aprendizagens ativas na educação em plena era da comunicação. Revista Metaliguagens. nº.4, p.64-77, 2015.

EICKHOLT, Jesse. Barriers to active learning for computer Science. Computers and Society. Disponível em: https://arxiv.org/abs/1808.02426v1. Acesso em: 26 ago. 2018.

EZENWABASILI, Mariana. Como as diferentes gerações aprendem. Ensino Superior, dez. 2016. Disponível em: http://www.revistaeducacao.com.br/como-diferentes-geracoes-aprendem/. Acesso em: 05 jun. 2018.

FREEMAN, S. et al. Active learning increases student performance in science, engineering, and mathematics. Proceedings of the National Academy of Sciences, v. 111, n. 23, p. 8410-8415, 12 mai. 2014.

GARNICA, L. A.; TORKOMIAN, A. L. V. Gestão de tecnologia em universidades: uma análise do patenteamento e dos fatores de dificuldade e de apoio à transferência de tecnologia no Estado de São Paulo. Gestão \& Produção, v. 16, n. 4, p. 624-638, dez. 2009.

HOLLAND, J.; HOLLAND, J. Implications of Shifting Technology in Education. TechTrends, v. 58, n. 3, p. 16-25, 21 abr. 2014. 
LOBO, A. S. M.; MAIA, L. C. G. O uso das TICs como ferramenta de ensino-aprendizagem no Ensino Superior / Use of technologies of information and knowledge as teaching-learning tools in higher education. Caderno de Geografia, v. 25, n. 44, p. 16-26, 30 jul. 2015.

LÓPEZ, M. J.; TRONCON, L. E. A. Capacitação e desenvolvimento docente - aspectos gerais. Medicina (Ribeirão Preto. Online), v. 48, n. 3, p. 282, 8 jun. 2015.

MACEIRA, Teresa E. \& DANITTA A. Wong. "Beyond Passive Learning: Utilizing Active Learning Tools for Engagement, Reflection, and Creation." Mobile Technology and Academic Libraries: Innovative Services for Research and Learning, edited by Robin Canuel and Chad Crichton for ACRL, Association of College and Research Libraries, 73-89, 2017.

MOLESWORTH, M.; NIXON, E.; SCULLION, R. Having, being and higher education: the marketization of the university and the transformation of the student into consumer. Teaching in Higher Education, v. 14, n. 3, p. 277-287, jun. 2009.

PARMELEE, D. X.; AL-KADI, A. S. Team-Based Learning. In: An Introduction to Medical Teaching. Springer Netherlands, p. 69-76. 2014.

PERES, C. M.; SUZUKI, K. M. F.; AZEVEDO-MARQUES, P. M. DE. Recursos tecnológicos de apoio ao ensino na saúde. Medicina (Ribeirão Preto. Online), v. 48, n. 3, p. 224, 8 jun. 2015.

ROACH, T. Student perceptions toward flipped learning: New methods to increase interaction and active learning in economics. International Review of Economics Education, v. 17, p. 74-84, set. 2014.

ROCHA, G. J. DA; LISBOA, W. T. “Nós não vamos fugir disso!": tensão e expectativas em torno do telefone celular como recurso pedagógico no processo ensino-aprendizagem em São José, Santa Catarina. Educação e Cultura Contemporânea, v. 13, n. 32, 2016.

SÁTYRO, Natália; SOARES, Sergei. A infra-estrutura das escolas brasileiras de ensino fundamental: um estudo com base nos censos escolares de 1997 a 2005.Instituto de Pesquisa Econômica Aplicada (IPEA), 2007.

SOARES, Neto et al. A infraestrutura das escolas públicas brasileiras de pequeno porte. Revista do Serviço Público; Brasília Vol. 64, Ed. 3, 2013.

SOUZA, E. A. DE et al. A Review of the Use of Information Technology in Brazilian Schools from 2010 to 2014. International Journal of Information and Education Technology, v. 7, n. 4, p. 284-290, 2017.

TIMBANE, Sansão Albino; AXT, Margarete; ALVES, Evandro. O celular na escola: vilão ou aliado! Nuevas Ideas en Informática Educativa TISE, s.v., p. 768-773, chile 2015.

TSENG, S.-F. et al. Who will pass? Analyzing learner behaviors in MOOCs. Research and Practice in Technology Enhanced Learning, v. 11, n. 1, 8 abr. 2016. 
VIEIRA, M.; ALVES, E. D. G. Celular e sala de aula: dos limites às possibilidades. Anais do XXI Workshop de Informática na Escola (WIE 2015). Anais. In: XXI WORKSHOP DE INFORMÁTICA NA ESCOLA. Sociedade Brasileira de Computação - SBC, 26 out. 2015.

Disponível em: <http://dx.doi.org/10.5753/cbie.wie.2015.236>. Acesso em: 26 ago. 2018.

Recebido em: 04/09/2019

Parecer em: 28/10/2019

Aprovado em: 16/11/2019 